\title{
El problema de la desarticulación de conocimientos en la formación inicial de profesores de química
}

\author{
The problem of the separation of knowledge in the initial formation of professors of \\ chemistry
}

Omar León Manchego Y Luis Enrique Salcedo Torres

Universidad Pedagógica Nacional. omarleon@pedagogica.edu.co y salcedo@pedagogica.edu.co

\section{Resumen}

En el presente artículo se plantea una de las problemáticas que está afectando de manera importante la formación inicial de profesores de ciencias y particularmente la formación inicial de profesores de química (FIPQ). Se trata de la desartiaulación entre los conocimientos químico y psico-socio-pedagógico enmarcada en el denominado modelo sumativo el cual prevalece en los programas de formación docente, en oposición a la reconciliación integradora necesaria para que ocurra un aprendizaje significativo.

Ante la ausencia de propuestas que ayuden a superar esta problemática sugerimos la implementación de estrategias de enseñanza como las basadas en la resolución de problemas químicos que posibiliten la construcción y a la vez la integración del conocimiento químico y el conocimiento psico-socio-pedagógico. A este conocimiento construido e integrado lo denominamos aquí Conocimiento Pedagógico del Contenido Químico (CPCQ)

Palabras clave

Desarticulación de conocimientos, Conocimiento Pedagógico del Contenido Químico, Resolución de problemas químicos, Formación inicial de profesores.

\section{Abstract}

In this article is presented one of the problems that is affecting in an important way the initial formation of science teachers and particularly the initial formation of chemistry teachers (IFCT). It is the separation among the knowledge chemist and psico-partner-pedagogic framed in the denominated model sumativo which prevails in the programs of educational formation, in opposition to the necessary integrative reconciliation so that happens a significant learning.

For the absence of proposals that help to overcome this problem we suggest the implementation of teaching strategies based on the resolution of chemical problems that facilitate the construction and at the same time the integration of the chemical knowledge and the psicopartner-pedagogic knowledge. To this built and integrated knowledge the denominate here Pedagogic Knowledge of the Chemical Content (PKCC)

\section{Keywords}

Separation of knowledge, Pedagogic Knowledge of the Chemical Content, Resolution of chemical problems, professors' initial Formation 


\section{Introducción}

Las investigaciones recientes en didáctica de las ciencias se han centrado principalmente en el desempeño de los profesores en ejercicio y en formación inicial toda vez que la enseñanza es uno de los principales factores que influye en el aprendizaje de los estudiantes. En este contexto las investigaciones señalan la desarticulación de conocimientos como problemática relevantes que requiere ser superadas en el proceso formativo de los profesores para favorecer el aprendizaje significativo. Para comenzar a superar esta problemática es importante que los PQFI tengan la oportunidad de vivenciar estrategias de enseñanza diferentes a las de transmisión-asimilación de conocimientos, como las basadas en la investigación, de modo que se favorezca la participación activa en la reconstrucción (Vilches y Gil, 2008) y la integración de conocimientos.

Lo que se persigue es que en los programas de formación docente se logre la construcción e integración de los conocimientos (químico, pedagógico, didáctico, psicológico, epistemológico, sociológico), superando la tendencia a estudiar los cursos disciplinares separados de los cursos psicopedagógicos y a centrar la enseñanza en la transmisión de los contenidos de la materia a enseñar o en la preparación psicopedagógica general.

Como la problemática descrita ha sido considerada relevante en la formación inicial de profesores de ciencias (Furió, 1994, Canascosa, et al, 2008) es preciso en el caso de la FIPQ comenzar por implementar estrategias de enseñanza como las basadas en la resolución de problemas químicos que propicien la construcción conceptual y procedimental y la integración de los conocimientos químico y psico-socio-pedagógico, construyendo Conocimiento Pedagógico del Contenido Químico (CPCQ).

\section{Desarticulación entre los conocimientos disciplinar, pedagógico, didáctico, psicológico, filosófico, sociológico.}

El problema de la desarticulación de conocimientos en la FIPQ está asociado con el "modelo sumativo“" (Furió, 1994) que se sigue en la mayoría de programas de formación docente y que ha sido objeto de críticas en las dos últimas décadas porque no favorece una formación docente de calidad. En este modelo, la formación de los futuros profesores se asume como la simple suma de una formación química (cursos de química) por una parte y una formación psicopedagógica (cursos de pedagogía, didáctica, psicología, filosofía, sociología) por otra.

Varios autores se han referido al problema de la desarticulación de conocimientos en la formación inicial de profesores. Shulman, (1986) y Furió y Gil, (1989), ya señalaban la existencia de dos mundos desconectados en que por una parte los profesores adquieren los conocimientos básicos o del contenido a través de cursos disciplinares y por otra parte, la formación psicopedagógica corresponde a cursos que toman sobre educación a nivel general. Esta desarticulación de conocimientos se presenta tanto en programas de FIPQ que ofrecen de manera simultánea cursos de química y aursos de formación psicopedagógica (p.e. programa para la formación inicial de profesores de química de la UPN) como en aquellos programas en los que se ofrece primero los cursos de química y al final los cursos de formación psicopedagógica.

Para Calderhead (1986) y Penick y Yager (1988), uno de los principales problemas en la formación de los profesores no es tanto desarrollar el conocimiento por parte de los alumnos en cada una de los cursos que constituyen el proceso formativo, sino, facilitar a los profesores en formación la integración de los conocimientos. Se ha considerado (Galluzzo, 1990; Ball, 2000) que la separación tradicional entre el conocimiento de la materia a enseñar y el conocimiento 
pedagógico ha sido una de las principales características de los programas de formación docente del último siglo.

En otros estudios (McDermott, 1990; Shulman, 1992; Pesa y Cudmani, 1998) se señala la dificultad que tienen los profesores en formación o en ejercicio para integrar los conocimientos de la materia a enseñar con los conocimientos psicopedagógicos. Ante esta situación se ha planteado reiteradamente (Dumás-Carré et al., 1990; Furió, 1994, Camicer 1998, Guisasola et al., 2001; Carrascosa, et. al., 2008) la necesidad de buscar la integración de los diversos conocimientos que conforman los programas de formación docente.

Dentro de las propuestas que tienen que ver con la integración del conocimiento de la materia a enseñar y el conocimiento psicopedagógico, destacamos la del conocimiento pedagógico del contenido -CPC- (Shulman, 1987) y la de la didáctica de las ciencias y formación inicial del profesorado (Furió y Gil, 1989; Carrascosa, et al., 2008). No obstante, para el caso de la FIPQ estas propuestas son insuficientes porque se basan en el estudio del CPC pero en profesores en ejercicio, conceden mayor importancia a los contenidos conceptuales que a los contenidos procedimentales y proponen el estudio de cursos de didáctica al final del plan de estudios suponiendo de esta manera la integración espontánea de conocimientos. Frente a esta situación proponemos una enseñanza basada en la resolución de problemas químicos para el desarrollo del CPCQ que implicaría la integración de los diversos conocimientos en el proceso de formación docente a medida que se estudian los cursos de química.

\section{Enseñanza de la química basada en la resolución de problemas químicos y desarrollo de conocimiento pedagógico del contenido químico}

Ante la necesidad de una formación para mejorar la calidad de la enseñanza, se requiere que más allá de construir conocimientos conceptuales de química y de conocer teorías generales sobre pedagogía, el futuro profesor de química construya conocimiento químico conceptual y procedimental y que lo integre con el conocimiento psico-socio-pedagógico. A este conocimiento integrado lo denominamos Conocimiento Pedagógico del Contenido Químico (CPCQ).

Una enseñanza basada en la resolución de problemas químicos en el contexto de la investigación permitirá al futuro profesor vivenciar procesos de producción del conocimiento químico, analizar sus implicaciones para la enseñanza y elaborar propuestas de enseñanza basadas también en la resolución de problemas químicos, construyendo de esta manera CPCQ. Esto implica orientar a los futuros profesores de química en: la interpretación y formulación problemas, la emisión de hipótesis, la identificación de variables, el diseño de procedimientos/montajes o dispositivos, la obtención y análisis de resultados, la socialización de los resultados y del trabajo realizado, el planteamiento de nuevos problemas, el análisis de cada uno de estos procesos en el contexto de la enseñanza de la química y la elaboración y sustentación de estrategias de enseñanza basadas en la resolución de problemas químicos por parte de los profesores de química en formación inicial.

Con base en la naturaleza de la investigación química y de conformidad con los aportes derivados de investigaciones sobre CPC, didáctica de las ciencias y formación inicial del profesorado, proponemos incluir en el CPCQ los siguientes aspectos: las finalidades de la enseñanza de la química, las ideas previas y dificultades de aprendizaje de los estudiantes sobre un determinado tópico de la química, los conceptos y procedimientos de la química, las estrategias de enseñanza basadas en la resolución de problemas químicos a partir de la transformación de contenidos en situaciones problemáticas y los criterios de evaluación en el contexto de la resolución de problemas. 
En el diagrama (Anexo 1) se representan los aspectos del CPCQ que los profesores de química en formación inicial podrían construir a través de los procesos de resolución de problemas químicos en el contexto de la investigación química.

Finalidades de la enseñanza de la química

La investigación química ha de contribuir a la solución de problemas relacionados con las sustancias químicas, no obstante, el campo espeáfico y la naturaleza de los problemas que se plantean depende de las creencias, actitudes, necesidades, intereses y políticas de los químicos que conforman los grupos de investigación.

La educación en química por su parte, debe contribuir al proceso formativo de nuestros jóvenes, esto es, al desarrollo de lo que en el ser humano es posible de mejorar. Así por ejemplo, el desarrollo de la creatividad, la crítica, la autonomía, la capacidad para resolver problemas, el trabajo colectivo, el ejercicio de la ciudadanía, ser un profesional reconocido, aportar a la solución de problemas en su entorno local y global. Cada vez toma mayor importancia la idea de educación cientúfica para la ciudadanía en contraposición a la tradicional educación propedéutica entendida como la preparación requerida para continuar estudios espećficos y el desarrollo de la conciencia por la preservación del ambiente. De acuerdo con Furió et al. (2001) y Vildhes y Gil (2008), a través de la enseñanza de la química se debería preparar a los futuros ciudadanos y entre ellos por supuesto a los futuros cient́ficos para que adquieran valores democráticos y conciencia de respeto y cuidado del medio en una educación orientada al desarrollo sostenible del planeta. A través de la enseñanza de la química es posible desarrollar habilidades propias del dominio afectivo tales como la capacidad de emitir juicios de valor, el respeto por las ideas de los demás, la capacidad para trabajar en grupo y por lo tanto la tolerancia y convivencia social (Cárdenas, 1998).

Concretamente, un profesor de química auando enseña un concepto químico no solo debería preocuparse por el aprendizaje de ese concepto por parte de sus estudiantes sino, por el impacto que su enseñanza tendrá en la constitución de un sujeto autónomo, ético, crítico, democrático, político; en una preparación que les permita participar colectivamente en la solución de los problemas con los que se enfrenta la sociedad de la que forman parte.

Ideas previas y dificultades de aprendizaje

De la misma manera que los paradigmas de la química influyen y orientan todos los procesos para la construcción de nuevo conocimiento químico y en especial la identificación y formulación de problemas y la emisión y fundamentación de hipótesis, las ideas previas que tienen los alumnos influyen también de manera favorable o desfavorable en el proceso de aprendizaje.

Gil y Ramírez (1990) señalan, que en la emisión de hipótesis los estudiantes regularmente expresan ideas "erróneas", pero que esto lejos de ser algo negativo, se constituye en una manera eficaz de explicitar las ideas previas de los estudiantes.

El concepto de ideas previas constituido en línea de investigación didáctica, se origina en la propuesta de Ausubel (1978) cuando plantea que el factor que mas influencia tiene en le aprendizaje es lo que el alumno ya sabe y que hay que averiguar qué es y enseñar de acuerdo con ello.

En general las ideas previas y las dificultades de aprendizaje de los estudiantes sobre el conocimiento químico están relacionadas con lo que significa para ellos la realidad externa. Entre los estudiantes es común una visión realista ingenua pensar que cualquier "hecho real" es la imagen directa que detectan nuestros sentidos (Pozo y Gómez, 1998). Para muchos estudiantes, la realidad del mundo natural concuerda con las percepciones sensoriales que quedan impresas directamente en la mente del sujeto, sin tener en cuenta que dichas percepciones dependen de lo que ya existe en ella (Johnstone et al., 1994). Se comprende así que los estudiantes pongan en duda la materialidad de aquellos estados de la materia que son dificilmente perceptibles, como en 
el caso de los gases (no se ven, no se tocan, no "pesan"). De esta manera, gran parte de las generalizaciones erróneas que cometen los estudiantes, se basan en la aplicación de razonamientos basados en el sentido común (Driver, 1985; Pozo y Gómez, 1998; Viennot, 2001) sobre el mundo natural al nivel microscópico de descripción de la materia. Es decir, para ellos el mundo de los átomos, moléculas, redes iónicas, etcétera, es el mismo mundo macroscópico de los materiales y las sustancias pero en diminuto. Según Talanquer, (2004) existe la tendencia a trasladar las propiedades del mundo macroscópico a los modelos microscópicos (los átomos se expanden de la misma manera que un sólido se expande al calentarlo), y a dar realidad concreta a estos modelos (los enlaces químicos son pequeños tubos conectores que pueden romperse). Los estudiantes no comprenden que existen distintos niveles de descripción de la materia en íntima relación: por una parte, el nivel macroscópico de las sustancias con sus propiedades y cambios y, por otra, el nivel microscópico de aquellas mismas sustancias que se modelan en química a base de átomos, iones 0 moléculas. Es con estas últimas entidades elementales que tienen sus propias propiedades e interacciones, con las que intentamos explicar unitariamente la estructura de la materia y los cambios químicos que observamos en la naturaleza.

Construcción de conocimiento conceptual y procedimental de la química

La construcción de conocimiento químico conceptual está asociada a la construcción de conocimiento procedimental. Gil y Paya (1991) y De Pro Bueno (1998) señalan que los procesos no son independientes de los contenidos.

El cambio conceptual (Posner, Strike, Hewson y Gerzog, 1982) en química consiste, en la modificación de las ideas previas de los alumnos para sustituirlas por los conceptos aceptados por la comunidad de químicos. No obstante, como señala Gil (1993), no es posible el cambio conceptual si este no se encuentra asociado a un cambio metodológico, que permita a los alumnos superar las formas de pensamiento "de sentido común" y abordar los problemas con una orientación cientúfica.

El cambio procedimental o metodológico consiste en el conocimiento que se construye y se infiere a partir del desarrollo de habilidades para observar, medir, identificar problemas, emitir hipótesis, diseñar experimentos, organizar y analizar datos, socializar resultados. También se induyen (Cárdenas, 1998) las habilidades para la adopción de procedimientos e instrumentos adecuados junto con los respectivos parámetros de seguridad, la capacidad para seleccionar los intervalos necesarios en una escala durante la realización de mediciones de una propiedad de la materia y el establecimiento de las variables sobre las cuales se debe recoger información.

Estrategia de enseñanza basada en la resolución de problemas químicos a partir de la transformación de contenidos en situaciones problemáticas

La estrategia de enseñanza basada en la resolución de problemas químicos puede ser la oportunidad para construir conocimiento químico conceptual y procedimental, vivenciando procesos de producción de conocimiento químico. Para ello, es necesario trasformar los contenidos químicos en situaciones problemáticas que generen interés y provean las condiciones para que los estudiantes con la orientación del profesor se impliquen en procesos de investigación.

En la fase inicial de la resolución de problemas químicos es importante la orientación adecuada del profesor en ejercicio para que el profesor en formación inicial (estudiante) se implique en la comprensión y solución del problema. Aparecen aquí dos momentos importantes en la investigación del estudiante. Uno corresponde al proceso de comprensión cualitativa de la situación problemática y al deseo por darle solución y el otro al proceso de solución de la situación planteada.

Que el estudiante comprenda la situación problemática y la haga suya son condiciones fundamentales para que se comprometa en su solución. Cuando esto ocurre el estudiante se ve en la necesidad de activar sus conodimientos previos y si no le son suficientes solicitará o buscará la 
información pertinente. Si no existen conocimientos previos o no son activados, para el alumno no habrá problema (Pozo, et. al., 1994). La comprensión de la situación problemática también es una ocasión importante para identificar conocimientos previos y dificultades de aprendizaje del estudiante, permitir la generación de todas las preguntas posibles y poner en movimiento los modelos teóricos que sustentan la problemática en estudio. En esta ocasión es necesario que el estudiante con base en sus teorías pueda establecer las variables que influyen en la situación problemática planteada. Esto requiere que el estudiante recurra al uso de modelos sobre el fenómeno en estudio.

La comprensión de la situación problemática tiene un importante valor psicológico, por que, en la medida que el estudiante la entienda, puede constituirse en un reto para buscar por si mismo la solución o soluciones posibles al problema planteado. Esta implicación afectiva es necesaria para que él tome la decisión de reaurir a sus conceptos y métodos para dar solución a la situación planteada. Es aquí donde el problema propuesto por el profesor se convierte en problema para el estudiante. Sin esta condición de voluntad y deseo del estudiante no será posible la implicación cognitiva del mismo en el proceso investigativo.

La emisión de hipótesis o posibles respuestas al problema es la ocasión para que el estudiante presente sus puntos de vista, lo que se convierte en un importante instrumento para expresar y contrastar las ideas previas. Son las hipótesis las que focalizan y orientan la resolución del problema, las que indican los parámetros a tener en cuenta y los datos a registrar y son las hipótesis junto con el auerpo de conocimientos en que se basan las que permitirán analizar los resultados y todo el proceso de investigación.

La fase de resolución del problema corresponde al proceso mediante el cual el estudiante establece el camino o los posibles caminos para contrastar hipótesis y obtener así las mejores respuestas a la pregunta/problema objeto de estudio. Es aquí donde se hace necesario establecer una metodología coherente con las bases teóricas para hallar la solución de una manera sistemática. Este proceso conduce en la mayoría de los casos a la necesidad de plantearse la realización de algún experimento que permita tener evidencia empírica de las hipótesis planteadas. Es entonces cuando surge la necesidad de hacer uso del laboratorio, lo cual requiere de una planeación que en términos generales implica: precisar las condiciones del experimento, identificar los materiales y/o los reactivos que se requieren, identificar (y en ciertos casos diseñar) los montajes, equipos o dispositivos tecnológicos que permitan obtener los datos experimentales.

El diseño y realización del experimento es un buen medio para que el estudiante desarrolle la creatividad, aspecto que queda marginado cuando las prácticas son planteadas como la simple reproducción de técnicas ya conocidas. La necesidad de pensar en el diseño de procedimientos, montajes, dispositivos e instrumentos para recoger la información; obliga al sujeto a producir esquemas, dibujos, gráficos, etc., que representen los diseños adecuados para el control de variables y el registro de datos.

El proceso de plantear el experimento como un camino de contrastación de hipótesis se completa con la ejeación del experimento mismo, es decir, con toda la actividad que implica el manejo de la tecnología para lograr el control de las variables y la obtención de resultados confiables y válidos que permitan posteriormente realizar los análisis correspondientes, derivar las conclusiones pertinentes y volver sobre las bases teóricas que sirvieron de partida a todo el proceso investigativo.

Al culminar la investigación pueden surgir nuevas preguntas/problema que den inicio a otra investigación para ampliar y/o aplicar los conceptos vistos. Este proceso investigativo se ve enriquecido por los aportes de todos los estudiantes quienes a través de las discusiones de sus resultados van mejorando sus aprendizajes, y la manera de comunicar sus pensamientos. La 
realización de informes, de resúmenes, de mapas conceptuales y la exposición de los mismos contribuyen a los procesos de socialización del trabajo realizado y permiten generar en el aula un ambiente de producción intelectual que favorece el desarrollo de actitudes positivas hacia el aprendizaje de la química.

\section{Criterios de evaluación en una enseñanza basada en la resolución de problemas químicos}

Una evaluación coherente con la naturaleza de la estrategia de enseñanza basada en la resolución de problemas químicos requiere de un seguimiento continuo para orientar e impulsar el proceso de construcción de conocimientos. Los profesores de química en formación inicial han de poder contrastar sus producciones con las de otros equipos y a través del profesor en ejercicio (director del proceso investigativo) con el resto de la comunidad científica; y han de ver valorado su trabajo y recibir la ayuda necesaria para seguir avanzando o para rectificar si es necesario. Este tipo de evaluación formativa (Novak, 1982; Coll, 1987) coherente con las tareas de investigación química debe formar parte, de los procesos de enseñanza/aprendizaje de la química. Se trata de concebir y utilizar la evaluación como instrumento de aprendizaje que permita suministrar información adecuada a los alumnos y al propio profesor, contribuyendo a la mejora de la enseñanza.

\section{Conclusiones}

Hemos planteado que la desarticulación de conocimientos se constituye en una problemática relevante y vigente en la formación inicial de profesores.

En los programas de formación inicial de profesores de ciencias y de química en particular se deberían implementar estrategias didácticas en el proceso formativo que superen el transmisionismo centrado en los contenidos de química o de pedagogía para que los futuros profesores puedan adaptar las estrategias aprendidas a la enseñanza de la química.

El futuro profesor debería construir CPCQ representado en la integración de los conocimientos químico y psico-socio-pedagógico. En el CPCQ debería induirse: las finalidades de la enseñanza de la química, las ideas previas y díficultades de aprendizaje, los conceptos y procedimientos de la química, las estrategias de enseñanza basadas en la resolución de problemas químicos y los criterios de evaluación.

Conviene diseñar e implementar estrategias de enseñanza que favorezcan el desarrollo de CPCQ durante el proceso formativo de los futuros profesores, a medida que se estudian los aursos de química, para romper con la creencia de que los futuros profesores lograran integrar espontáneamente los conocimientos que se estudian de manera aislada en los cursos de química por una parte y de pedagogía general por otra.

Las estrategias de enseñanza basadas en la resolución de problemas químicos pueden servir de base para el desarrollo de CPCQ mediante la vivencia de procesos de construcción del conocimiento químico y el reconocimiento de las implicaciones que estos procesos tienen en la enseñanza y el aprendizaje de la química.

\section{Bibliografía}

AUSUBEL, D. (1978). Psicología Educativa. Un punto de vista cognoscitiva México: Editorial Trillas 
BALL, D. (2000). Bridging Practices: Intertwining Content and Pedagogy in Teaching and Learning to. Teach. Joumal of Teacher Education, 51 (3), 241- 247

CALDERHEAD, J. (1986). La mejora de la práctica de la dase: aplicaciones de la investigación sobre la toma de decisiones en la formación del profesorado, Actas del I Congreso Internacional sobre Pensamiento de los Profesores y Toma de Decisiones. Servicio de Publicaciones de la Universidad de Sevilla

CARDENAS, F. (1998). Desarrollo y evaluación de los procesos de razonamiento complejo en ciencias. Teané, Episteme y Didaxis: TED No. 3. pp. 53-68

CARNICER, J. (1998). El cambio didáctico en el profesorado de ciencias mediante tutońas en equipos cooperativos. Tesis Doctoral. Universidad de Valencia.

CARRASCOSA, J; MARTINEZ, J. FURIO, C. y GUISASOLA, J. (2008) ¿Qué hacer en la formación inicial del profesorado de ciencias de secundaria?. Revista Eureka, 5(2). pp. 118-133

COLL, C. (1987). Psicología y Curriaulum Barcelona: Laia.

DE PRO BUENO, A. (1998). ¿Se pueden enseñar los contenidos procedimentales en las clases de ciencias?. Enseñanza de las ciencias, 16(1), pp. 21-40

DRIVER, R. (1985). Children's Ideas in Science. Milton Keynes, UK: Open University Press.

DUMAS CARRE, A., FURIO, C. y GARRET, R. (1990). Formación inicial del profesorado en Francia, Inglatenra, Gales y España. Análisis de la organización de los estudios y nuevas tendencias. Enseñanza de las Ciencias, 8 (3), pp. 274-281.

FURIO, C. (1994). Tendencias actuales en la formación del profesorado en ciencias. Enseñanza de las ciencias, 12(2). pp. 188-199

FURIO, C. y GIL, D. (1989). La didáctica de las ciencias en la formación inicial del profesorado: una orientación y un programa teóricamente fundamentados. Enseñanza de las ciencias, 7(3). pp. 257-265

FURIÓ, C., VILCHES, A, GUISASOLA, J. y ROMO, V. (2001). Finalidades de la enseñanza de las ciencias en la Secundaria Obligatoria. ¿Alfabetización científica 0 preparación propedéutica?. Enseñanza de las Ciencias, 19(3). pp. 365- 376.

GALLZZO, G., AND CRAIG, J. (1990). Evaluation of preservice teacher education programs. In W. R. Houston (ed.), Handbook of Research on Teacher Education. New York: Macmillan. p.p. 599616

GIL, D. y GUZMÁN, M. (1993). Enseñanza de las Ciencias y la Matemática. Tendencias e Innovaciones. OEI para la Educación, la Ciencia y la Cultura. Editorial Popular. En: http://unw.campusoei.org/oeivirt/ciencias.htm/

GIL, D. y PAYA, J. (1991). Los trabajos prácticos en la enseñanza de la física y química. Un análisis crítico y una propuesta fundamentada. Tesis Doctoral. Universidad de Valencia.

GIL, D. y RAMIREZ, J. (1990). La resolución de problemas de física y química como investigación en la enseñanza media: un instrumento de cambio metodológico. Tesis Doctoral. Universidad Autónoma de Barcelona. 
GUISASOLA, J.; PINTOS, M. y SANTOS, T. (2001). Formación continua del profesorado, investigación didáctica e innovación en enseñanza de las ciencias, Revista Interuniversitaria de Formación del Profesorado. 41. pp. 207-222

JOHNSTONE, A., SLEET, R.J. Y VIANNA, J.F. (1994). An information processing model of leaming: its application to an undergraduate laboratory course in Chemistry, Studies in Higher Education, 19. pp. 77-87

MCDERMOTT, LC. (1990). A perspective on teacher preparation in physics - other sciences: the need for special science courses for teachers. American Journal of Physics, 58 (8), pp.734-742.

PENICK, J. y YAGER, R. (1988). Science Teacher Education: A program with a theoretical and pragmatic rationale, Joumal of Teacher Education, Nov-Dic, 59-64.

PESA, M. y CUDMANI, L (1998). La integración de saberes en la formación de formadores en ciencias. En JM Sánchez Jiménez, (Ed). Educación Científica, 55-70. Alcalá de Henares: Servicio de publicaciones de la Universidad de Alcalá.

POSNER, G.; STRIKE, K.; HEWSON, P; GERTZOG, W. (1982). "Acomodation of a scientific conception: Toward a theory of conceptual change", Science Education, 66(2), 211-227.

POZO, J. y GOMEZ, M. (1998). Aprender y enseñar ciencia. Del conocimiento cotidiano al conocimiento cient́fico. Madrid: Morata, pp. 281-288.

POZO, J.; PEREZ, M.; DOMINGUEZ, J.; GOMEZ, M y POSTIGO, Y. (1994). La solución de problemas. España: Aula XXI Santillana

SHULMAN, L. (1986). Those Who Understand: Knowledge Growth in Teaching. Educational Researcher. Vo.15, No. 2, pp. 4-14.

SHULMAN, L (1987). Knowledge and Teaching: Foundations of the New Reform, Harvard Educational Review, 57(1). 1-22

SHULMAN, L (1992). Renewing the Pedagogy of Teacher Education: The Impact of SubjectSpecific Conceptions of Teaching. Las Didácticas Específicas en la Formación del Profesorado (pp. 53-69), Santiago de Compostela: Tórculo Edicións.

TALANQUER, V. (2004). Formación docente: ¿Qué conocimiento distingue a los buenos maestros de química?. Educación Química, 15(1), 52-58.

VIENNOT, L. (2001). Reasoning in physics: the part of common sense Dordrecht: kluwer Academic Press.

VILCHES, A. y GIL, D. (2008). La sostenibilidad y el debate nudear, Revista Eureka sobre Enseñanza y divulgación de las Ciencias, vol. 5 (1), pp. 94-99. 


\section{ANEXO 1.}

ENSEÑANZA DE LA QUIMICA BASADA EN LA RESOLUCION DE PROBLEMAS QUIMICOS Y CONSTRUCCION DE CPCQ

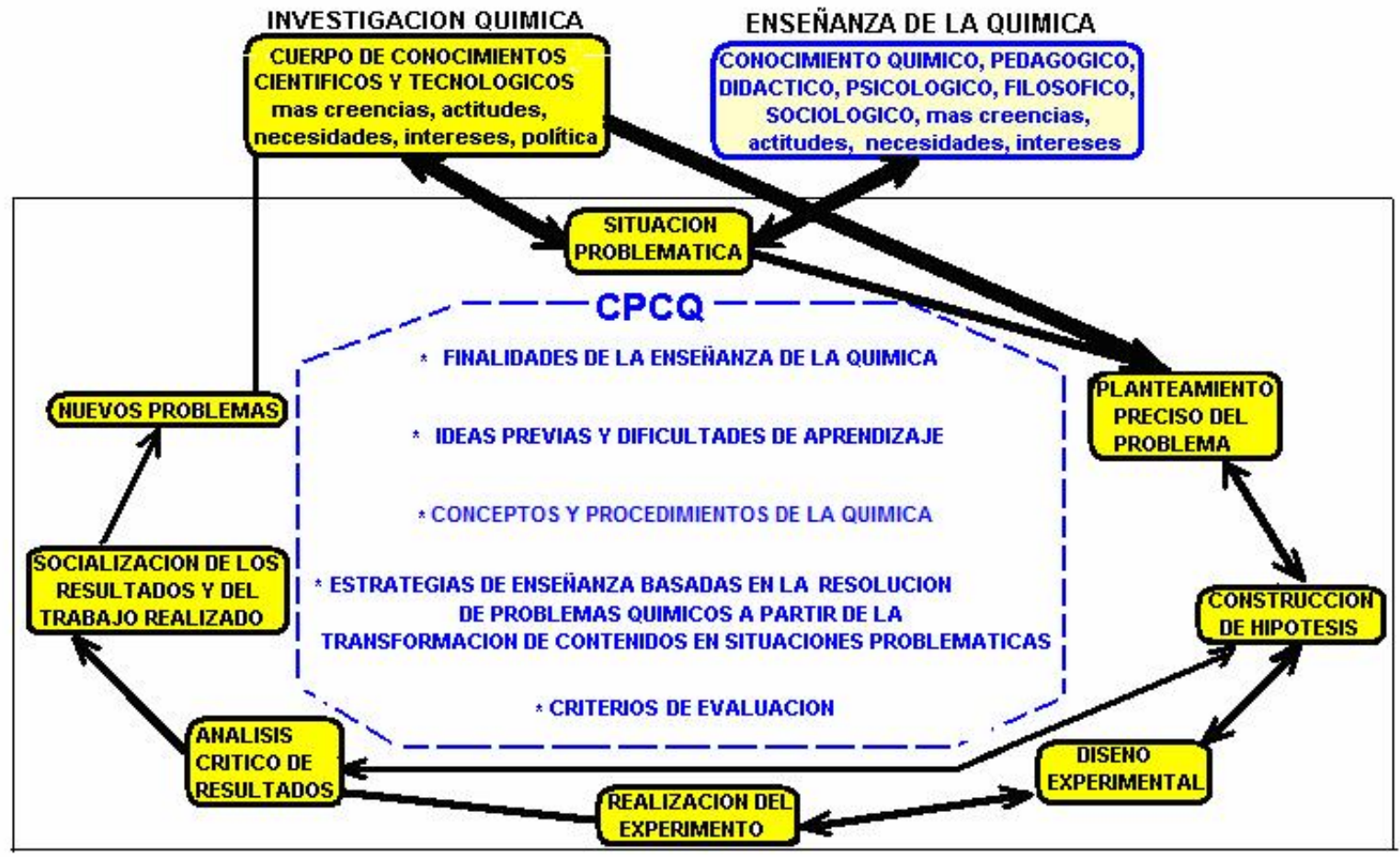

